\title{
KHK-Risikofaktor Stress im Beruf
}

\section{Eine hohe berufliche Beanspruchung erhöht auch bei Frauen das Risiko für kardiovaskuläre Komplikationen, wo- bei die traditionellen Risikofaktoren nur eine untergeordnete Bedeutung haben.}

- Die Daten stammen aus der Women's Health Studie: Bei Frauen, die im Gesundheitswesen tätig sind, überwiegend Krankenschwestern, wurde zwischen 1993 und 2007 die Wirkung von ASS und Vitamin $\mathrm{E}$ auf die Entstehung von kardiovaskulären Erkrankungen und Malignomen untersucht. Von 22086 im Mittel 57 Jahre alten Probandinnen gibt es Aussagen zur beruflichen Situation. Im Verlauf von zehn Jahren wurden 170 Herzinfarkte, 163 ischämische Schlaganfälle, 440 Revaskularisierungen der Koronargefäße und 52 kardiovaskuläre Todesfälle registriert.

Frauen mit hoher beruflicher Beanspruchung in Bezug auf Arbeitstempo, Herausforderung, Arbeitsumfang, Zeit- druck und konkurrierende Aufgaben hatten eine um 38\% höhere kardiovaskuläre Komplikationsrate als solche mit geringen Anforderungen, und zwar unabhängig davon, ob sie nach ihrem subjektiven Empfinden die Anforderungen bewältigen und kontrollieren konnten oder nicht. Besonders häufig waren nicht tödliche Infarkte und koronare Revaskularisierungen. Die Sicherheit des Arbeitplatzes, die in vier Kategorien von „sehr sicher" bis „sehr unsicher" eruiert wurde, war ohne Einfluss auf das kardiovaskuläre Langzeitrisiko.

\section{Kommentar}

Diese Ergebnisse stimmen mit denen aus Studien an Männern überein. Natürlich bleibt die Frage unbeantwortet, warum eine starke berufliche Beanspruchung kardiovaskuläre Krankheiten begünstigt. Aus mechanistischer Sicht könnte es einen indirekten Einfluss über Änderungen bei den klassischen Risikofaktoren und Verhaltensänderungen wie Rauchgewohnheiten oder depressive Verstimmungen geben, doch ist deren Einfluss auf maximal 11-26\% begrenzt. Deshalb werden als Ursache vor allem physiologische Stressreaktionen mit Dysregulation im hypothalamischen und autonomen System diskutiert, die direkt über einen Blutdruckanstieg, eine Destabilisierung atherosklerotischer Plaques und vermehrte Bildung von Zytokinen und Kortisol mit endothelialer Dysfunktion zur Insulinresistenz und metabolischen Störungen führen. Dass die Sicherheit des Arbeitsplatzes ohne Einfluss auf kardiovaskuläre Komplikationen bleibt, steht im Widerspruch zur Mehrzahl früherer Daten.

H. HOLZGREVE -

\section{Obstruktive Parotitis}

Ein 51-jähriger Mann stellte sich wegen seit drei Tagen bestehender Schmerzen, einer Schwellung der rechten Gesichtshälfte, Fieber und Mundtrockenheit in der Nothilfe vor. Bei der klinischen Untersuchung erkannte man eine präaurikulär gelegene rechtsseitige Schwellung mit einer Rötung der darüber liegenden Haut. Die Mundhöhle war trocken und zeigte eine Schwellung im Bereich des Ausführungsgangs der rechten Parotis, aus dem sich kein Speichel entleerte (Abb. A, Pfeil).

Bei den Laboruntersuchungen fielen eine Leukozytose von $21400 / \mu l$ mit $88 \%$ neutrophilen Granulozyten auf. In der Computertomografie des Gesichtsschädels stellte sich eine Entzündung der rechten Glandula parotis und des umgebenden Gewebes mit einer deutlichen Dilatation des Ausführungsgangs (Abb. B, roter Pfeil) sowie eine kleine umschriebenen Verkal-

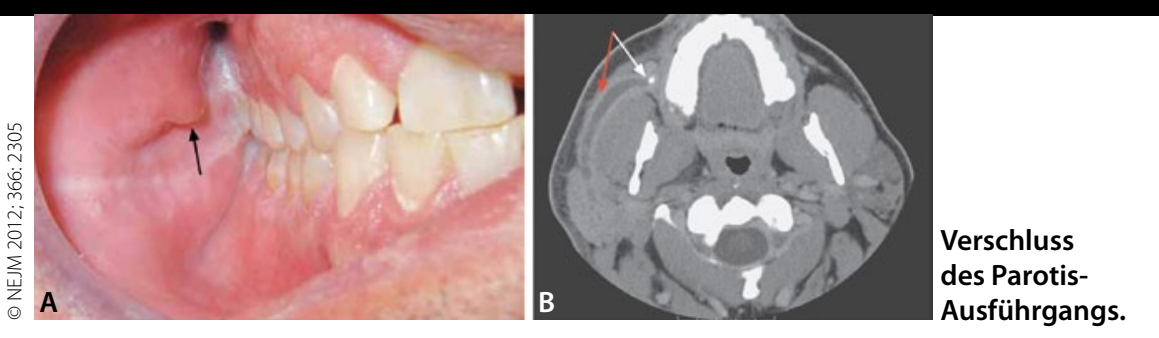

kung unmittelbar proximal der Gangöffnung dar (Abb. B, weißer Pfeil).

Ein Verschluss des Parotis-Ausführungsgangs aufgrund einer ödematösen Entzündung kann zur akuten Parotitis führen. Die Kombination aus Sekretstase, Veränderungen in der Zusammensetzung des Speichels und eine Infektion können zur Bildung von Speichelsteinen führen, die sich wegen des hohen Kalziumsgehalts häufig radiologisch darstellen lassen.

Die Behandlung besteht in der Entfernung der Gangsteine und einer unterstützenden antibiotischen Therapie, wodurch bei den meisten Patienten einem Rückgang der Symptomatik erzielt werden kann. Der hier vorgestellte Patient unterzog sich einer chirurgischen Entfernung des Steins. Zusätzlich wurde in den Gang ein Stent implantiert und der Patient antibiotisch behandelt. Hierunter besserten sich die Beschwerden zögerlich. In der Bakterienkultur war Streptococcus anginosus nachweisbar.

H. S. FÜESSL -

- S. M. Susarla und Z. S. Peacock

(Massachusetts General Hospital, Boston, MA) Obstructive parotitis. New Engl. J. Med. 2012; 366: 2305 